\title{
Light beam induced finger instability in a photosensitive liquid crystal cell
}

\author{
Ignacio Andrade-Silva $\odot^{*}$ \\ Institut Universitaire des Systèmes Thermiques et Industriels, Aix-Marseille Université, \\ CNRS, 13013 Marseille, France \\ Marcel G. Clerc $\odot,{ }^{\dagger}$ Gregorio Gonzalez-Cortes $\odot,{ }^{\ddagger}$ and Vincent Odent ${ }^{\S}$ \\ Departamento de Físicas and Millennium Institute for Research in Optics, Facultad de Ciencias Físicas y Matemáticas, \\ Universidad de Chile, Casilla 487-3 Santiago, Chile
}

(Received 20 April 2021; accepted 9 June 2021; published 21 June 2021)

\begin{abstract}
Interfaces limiting two phases in driven systems may exhibit rich dynamical behaviors. Optically induced phase transitions are of particular interest for the study of interfacial phenomena. Dye-doped liquid crystals offer an ideal scenario in which phase transitions can be induced by purely optical means. Light-driven interface dynamics and spatial structure have been partially unveiled. Here we report the emergence of fingerlike structures at the nematic-isotropic interface in a photoisomerization experiment. A reduced model reveals the instability origin, which is derived from a model for the dopant concentration and the order parameter. Analogously to the hydrodynamically triggered finger instability, the interdigital space follows a power law. When the cell is in the isotropic phase and the light turns off, transient foamlike and labyrinthine textures are observed. Numerical simulations reproduce the observed behaviors. Our findings open an avenue for optically addressed interfacial dynamics and shape tailoring of spatial textures, not only liquid crystals but also polymers.
\end{abstract}

DOI: 10.1103/PhysRevResearch.3.L022027

Interfaces are the physical borderline between two immiscible phases of matter. Depending on the nature of the states connected, interfaces can undergo spatial instabilities, exhibiting complex dynamical behaviors and displaying enthralling shapes. Interfacial phenomena can be found in multiple physical systems and have been a subject of study for decades. A paradigmatic example that has accompanied and intrigued humanity since its dawn is the spread of fire [1,2]. The interface instability is the result of an unbalance between expansive and limiting factors. Snowflake growth is perhaps the most famous case of interface instability leading to intriguing motifs due to heat diffusion limitations [3]. Interfacial instabilities have also been observed in metal deposition [4], where the limitation is the electrodiffusion; retinal vessel growth [5]; and bacterial colonies [6], where mass diffusion is the limiting factor. One of the most studied scenarios is viscous fingering, also known as finger instability, where fingerlike structures grow when a low-viscosity fluid is injected into a higher one, creating paths of lower resistance $[7,8]$. The fingering phenomenon was initially studied in molasses-water mixtures [9] and later

\footnotetext{
*ignacioandradesilva@gmail.com

†marcel@dfi.uchile.cl

‡regorio.gonzalez@ug.uchile.cl

§vincent.odent@saint-gobain.com
}

Published by the American Physical Society under the terms of the Creative Commons Attribution 4.0 International license. Further distribution of this work must maintain attribution to the author(s) and the published article's title, journal citation, and DOI. studied in a more controllable setup in which a fluid is in a Hele-Shaw cell while another immiscible fluid is injected [10]. Finger instabilities can be found in other contexts, such as in elastic buckling [11] and laser-induced implosions in cylindrical tubes [12]. All cited interfacial instabilities are spontaneously emergent or controlled by hydrodynamic injection, thermal, or electro-osmotic pressure [13].

In recent years, liquid crystal based optical systems have been shown to be of great interest for the study and tailoring of complex interfacial phenomena because of their high control due to light beam shaping techniques $[14,15]$. Liquid crystals are a state of matter that is allowed to flow like conventional liquids while having properties usually attributed to solids like birefringence and can exist in a multitude of phases with varying degrees of order $[16,17]$. Depending on the mechanism used to trigger the phase transitions between the mesophases, liquid crystals are classified as thermotropic, lyotropic, or polymeric, among others [18]. For optical applications, it is desired to control either the material's physical properties or its light response. By incorporating a photoresponsive dopant (typically, an azo dye), the coupling between the liquid crystal and light can be amplified [17,19,20]. Upon illumination, the azo dye undergoes a photoisomerization process, inducing a phototropic host-guest phase where the nematic liquid crystal changes to an isotropic state [21,22]. This phenomenon is known as the Jánossy effect. The nematic-isotropic optically induced phase transition is of particular interest for birefringence control. This opens the possibility of optically operated devices [23-25]. Since the discovery of the Jánossy effect, most advances have focused on creating new mixtures by improving the azo dye molecules' response and 
characterizing their linear and nonlinear responses. Lightdriven interface dynamics between coexisting mesophases have remained concealed except for some simple configurations. Propagation of locally flat nematic-isotropic interfaces has been observed in cells with planar anchoring [15] and soliton propagation in dye-doped liquid crystal cells [26]. Recently, the emergence of patterns in dye-doped liquid crystal cells due to differences between the cis-isomer diffusion and molecular orientation timescales [27-29] and light-induced motion of dye-doped liquid crystal droplets [30-34] was reported.

This Letter aims to describe the emergence of light-induced fingering instability of a nematic-isotropic interface in a liquid crystal photoisomerization experiment while having the possibility of controlling propagation speed, finger length, and average interdigital spacing by optical means. Furthermore, the system shows complex foamlike and labyrinthine textures when the process is reversed by decreasing the light intensity. We propose a phase-field model that reproduces all the observed behaviors based on reaction-diffusion equations for the liquid crystal order parameter and the cis-state dye concentration. These results open the avenue for developing new optical techniques for controlling liquid crystal and polymeric mixtures textures.

The experimental setup is depicted in Fig. 1(a). We used a dye-doped liquid crystal cell (DDLCC) consisting of a nematic liquid crystal mixed with methyl red dye and sandwiched between two polyvinyl alcohol-coated glass plates separated by $25 \mu \mathrm{m}$ thanks to boundary spacers. The liquid crystal is commercially available E7 (by Instec), with elastic constants $K_{1}=11.2, K_{2}=6.8$, and $K_{3}=18.6\left(\times 10^{-12} \mathrm{~N}\right)$ and dielectric constants $\epsilon_{\|}=18.96$ and $\epsilon_{\perp}=5.16$. The dye corresponds to a methyl red dye (2- $N, N$-dimethyl-4aminophenyl) which is mixed at a concentration of $0.5 \mathrm{wt} \%$ as solid crystal powder with the E7 liquid crystal. The mixture is heated to enhance mixing and homogenization of the mixture. The low concentration of dye ensures that the resulting material is a liquid crystal at room temperature and elastic property preservation while increasing the optical coupling. Once the mixture is homogeneous, it is injected between two polyvinyl alcohol coated glasses separated by $25 \mu \mathrm{m}$ boundary spacers and rubbed to favor planar alignment and orthogonal to each other to create a planar twisted alignment cell [35]. The surface area of the cell is about $4 \mathrm{~cm}^{2}$. This configuration favors the dopant molecules arranging in different orientations following the liquid crystal molecular orientation in the nematic phase, ensuring the necessary coupling with the light crossing the sample. A DDLCC region is illuminated with a Gaussian light beam from a frequency-doubled $\mathrm{Nd}^{+3}: \mathrm{YVO}_{4}$ laser with a wavelength of $532 \mathrm{~nm}$. The wavelength was selected to be in the absorption band of the methyl red dye [36]. Light absorption of the DDLCC has been measured which increases the sample temperature up to $38^{\circ} \mathrm{C}$ under similar experimental conditions, $22^{\circ} \mathrm{C}$ below the thermotropic nematic-isotropic transition [14]. Hence, under these thermal conditions, the system is in its nematic mesophase. The laser is expanded and spatially filtered using a Kepler telescope with a magnification of $5 \times$ and a pinhole placed in the focal point of the telescope. A double Glan Calcite polarizer is used to ensure the polarization of the beam. A single Glan Calcite polarizer or extinction
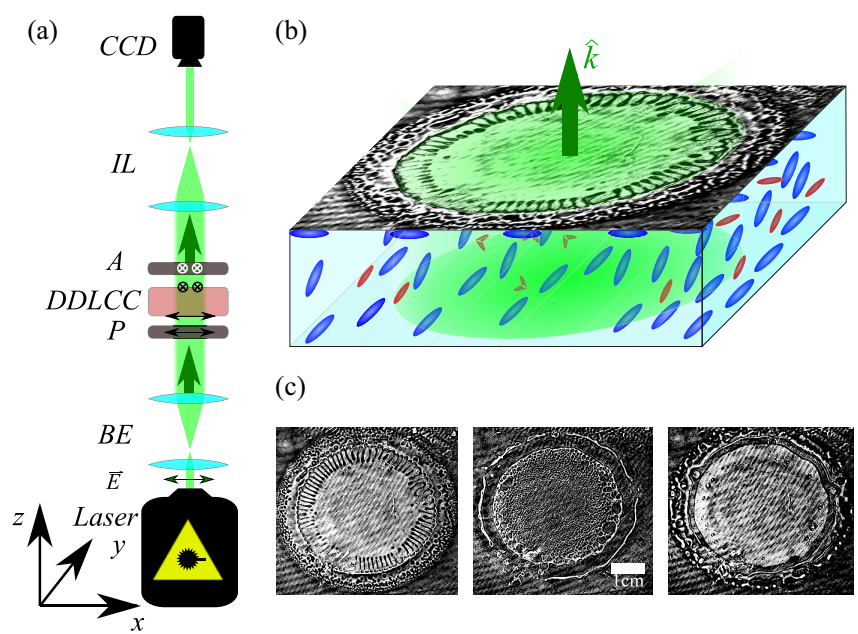

(c)
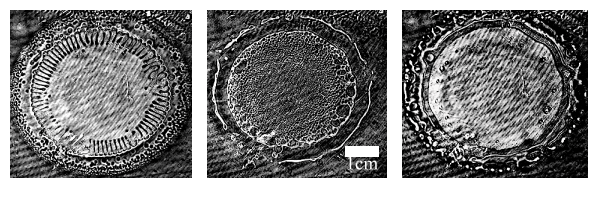

FIG. 1. Experimental setup and interface dynamics. (a) The system is irradiated by a frequency-doubled $\mathrm{Nd}^{+3}: \mathrm{YVO}_{4} 532 \mathrm{~nm}$ green laser. A pair of lenses (BE) is used to expand the laser beam. A polarizer $P$ is placed to add extra laser power control. The dye-doped liquid crystal cell (DDLCC) is placed after the first polarizer $P$ following the beam propagation direction. The DDLCC has twisted anchoring. An analyzer $A$ is placed to sample the birefringence of the DDLCC. The horizontal and entering the plane arrows indicate the polarization directions of the electric field, polarizer, anchoring, and analyzer. Namely, the polarizer $P$ and analyzer $A$ are oriented on the $x$ and $y$ axes, respectively. Imaging lenses (IL) and a CCD camera are used to acquire the images. (b) Schematic representation of the host-guest phototropic phase transition in the DDLCC. The Gaussian beam profile induces a spatial distribution of the photoisomerization rate in the guest dye methyl red, producing a decrease in the host liquid crystal nematic order. (c) Snapshots of the dye-doped liquid crystal textures obtained for different conditions. The left snapshot is obtained for green laser irradiation of $700 \mathrm{~mW}$ when the fingers reach their maximum extension, the central snapshot is for $10 \mathrm{~mW}$ irradiation starting from an isotropic bubble, and the right snapshot is an isotropic stationary bubble for irradiation of $700 \mathrm{~mW}$.

polarizers can be used instead. The DDLCC cell with planar twisted anchoring is placed perpendicular to the optical axis. An analyzer crossed polarizer is placed to filter the light that passes through the liquid crystal cell. The analyzer corresponds to a Glan Calcite polarizer. A Kepler telescope with a magnification of $0.25 \times$ is used to record the light with a monochrome CCD camera with $1024 \times 768$ resolution recording at 20 frames per second. The DDLCC was subjected to input powers from 300 up to $700 \mathrm{~mW}$. All experimental observations were made at room temperature. When the cell is not illuminated, the sample is in a homogeneous twisted nematic configuration, and the methyl red is in the trans isomer state, which tends to align with the orientational order of the liquid crystal molecules [37]. When illuminating, the methyl red undergoes isomerization, changing from a trans-isomer to a cis-isomer due to photoexcited molecular torque [38] [see Fig. 1(b)]. Cis-isomer molecules induce local disorder on the liquid crystal because of its inability to align with the nematic director $[17,27]$. For high illumination intensity, the system undergoes a host-guest phototropic transition where the nematic phase locally changes to an isotropic liquid [39]. 


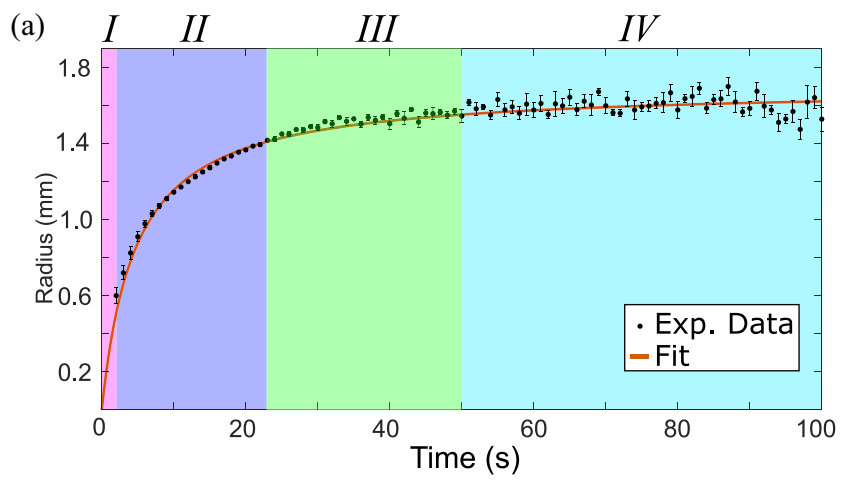

(b)

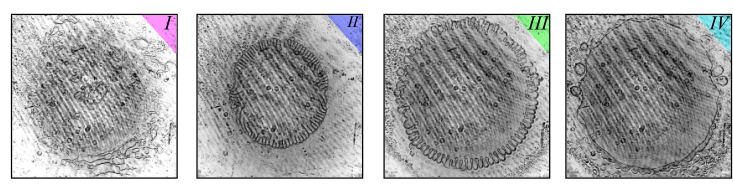

(c)

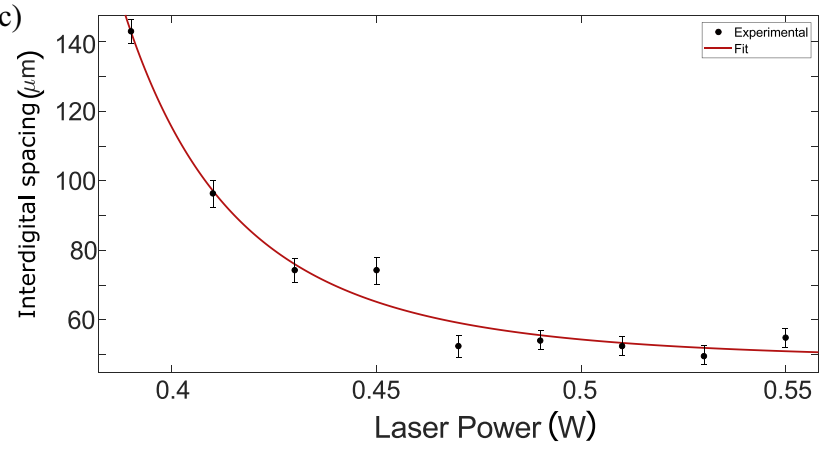

FIG. 2. Experimental features of the spatial transversal instability. (a) Temporal evolution of the mean radius of the isotropic region. (b) The dynamics can be divided into four stages: (I) nucleation, (II) expansion, (III) saturation, and (IV) steady state. (c) For different light intensities, different average interdigital spacing emerges. The interdigital spacing is measured as the distance between two fingers when the isotropic bubble reaches its maximum radius (the transition from region II to region III. A power law with measured critical exponent -3.27 is fitted.

The system displays rich, diverse dynamics, from which we have identified three main behaviors [see Fig. 1(c)]: finger instability, foamlike texture, and labyrinthine texture. We first describe the behavior of the system upon illumination. It can be divided into four stages: nucleation, expansion, saturation, and the steady state [see Fig. 2(a)]. First, the nematic-isotropic transition occurs through the nucleation of isotropic bubbles into the nematic liquid crystal close to the center of the illumination zone. These bubbles are created due to inhomogeneities in the cell, which trigger the photoisomerization front in several places. The bubbles coalesce rapidly to form a single isotropic expanding bubble that invades the nematic liquid crystal following the dynamics dictated by the Gaussian light beam. Once a single bubble of the isotropic state is formed, the interface turns unstable, developing a spatial modulation. Instabilities of a moving nematic-isotropic interface-driven were reported [40,41]. This modulation gives rise to fingerlike structures that grow towards the isotropic state: the fingertips propagate slower than their base until they reach a maximum length (see the temporal sequence in Fig. 2(b). The number of fingers depends on the light intensity. Indeed, the finger average interdigital spacing decreases following a power law as the light intensity increases, as shown in Fig. 2(c). Analogous behavior is also observed in the Saffman-Taylor experiment, where the finger periodicity decreases with the capillary number [42]. This decrease suggests that in our experiment, a similar mechanism takes place. Namely, the interface follows the least hydrodynamic resistance path between two immiscible fluids, which are the nematic and isotropic phases. Later, the interface stops at the position where both nematic and isotropic phases are equally favorable. This position corresponds to the Maxwell point [15]. Subsequently, fingers shrink until the interface becomes circular. At this point, no further dynamic is observed, and the front becomes motionless.

Coupling between liquid crystal order parameter $S$ and the cis-state photoisomerizable molecule concentration $c$ has been used previously to model host-guest phototropic transitions on dye-doped liquid crystal cells with planar and twisted anchoring $[27,28]$. Close to a Turing spatial instability the following phase-field reduced model can be derived for the order parameter in a DDLCC subjected to Gaussian irradiation [27],

$$
\partial_{t} u=\eta(r)+u-u^{3}+D(r) \tilde{\nabla}^{2} u-\nabla^{4} u,
$$

where $u$ is related to the order parameter of the liquid crystal. Notice $u \approx 1(u \approx-1)$ accounts for the nematic (isotropic) phase. $\eta(r)=\alpha\left(C_{0}+C_{1} e^{-r^{2} / \omega^{2}}\right)$ is an inhomogeneous bifurcation parameter modeling preference of the material to have a nematic (isotropic) phase for $\eta>0(\eta<0)$ [15,27]. The parameters $C_{0}$ and $C_{1}$ are determined by the light intensity [27]. Hence, the bifurcation parameter inherits the laser beam intensity structure, having a Gaussian profile with respect to the radial coordinate $r . \alpha$ is a constant parameter tuning the coupling between the order parameter and the light intensity. $\tilde{\nabla}^{2}$ is an anisotropic diffusion operator with respect to the radial and the angular coordinate $\theta$ which reads

$$
\tilde{\nabla}^{2}=\left(\frac{\partial^{2}}{\partial r^{2}}+\frac{1}{r} \frac{\partial}{\partial r}+\lambda \frac{1}{r^{2}} \frac{\partial^{2}}{\partial \theta^{2}}\right) .
$$

$D(r)$ is an inhomogeneous diffusion coefficient. When $D<$ 0 , a Turing instability takes place, triggering pattern formation. Pattern formation is seen only on the nematic state, not in the isotropic one, for which we introduced a spatial dependence

$$
D=D_{H}+D_{I} \tanh \left(\frac{r-r_{0}}{p}\right),
$$

which allows the control of the pattern formation zone. $\lambda$ is an angular anisotropy, which controls the fingering instability. For $\lambda=1$, we obtain the conventional Laplacian operator. The intrinsic anisotropy of the liquid crystal matrix and the Gaussian illumination is the origin of the anisotropy, which favors the cis-state concentration transport in the radial direction. Similar models have been used for the host-guest phototropic transition on dye-doped liquid crystal cells with planar anchoring cells $[14,15]$ and the emergence of stripe patterns in a cell with twisted anchoring [28]. The partial differential equation (1) is solved using a finite difference scheme 
(a)

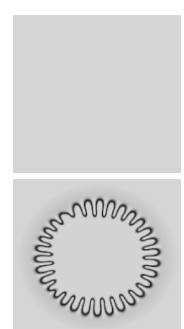

(b)

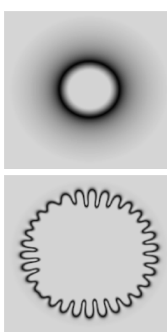

$\mathrm{C}_{1}$
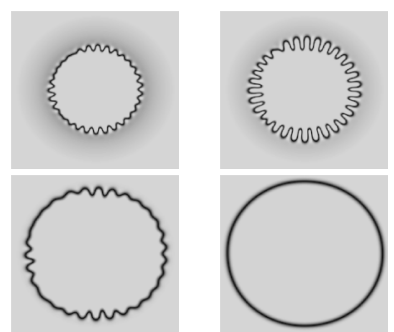

(c)

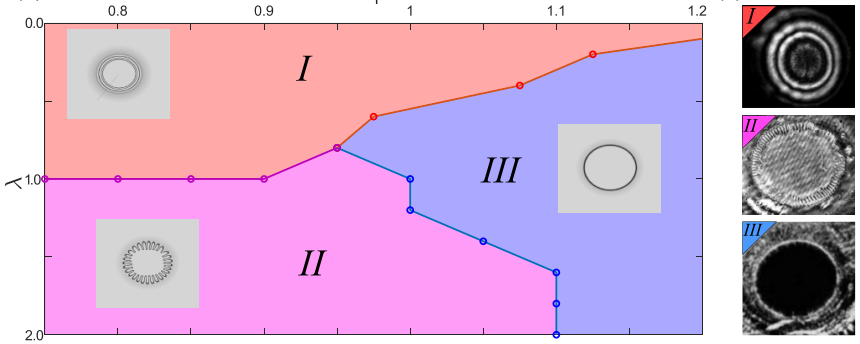

FIG. 3. Numerical simulations of equation Eq. (1). (a) Temporal sequence of the evolution of the order parameter $u$ showing finger instability (black stands for $u \approx 0$ ) for $C_{0}=-0.25, C_{1}=1.0$, $\omega=58, D_{H}=-0.6, D_{I}=1.9, r_{0}=105, p=40$, and $\lambda=1.5$ in a two-dimensional grid with $300 \times 300$ points. (b) Phase diagram in the parameter space $\left\{C_{1}, \lambda\right\}$ with the same values of the rest of the parameters as in (a). Regions I, II, and III account for concentric ring propagation, finger instability, and circular isotropic droplet propagation, respectively. (c) Typical experimental snapshots of the related phenomena for regions I, II, and III in the phase diagram.

in space that uses a centered stencil of seven grid points, where spatial derivatives are calculated using expansion up to order 4 in the reticle size. Time integration is performed using the Runge-Kutta algorithm with leading order 4. Figure 3(a) shows a temporal sequence of a typical simulation in which a nematic-isotropic interface develops a fingering instability as it propagates starting from a purely nematic initial condition. Varying parameters $C_{1}$ and $\lambda$, three different scenarios can be identified: concentric rings propagation, finger instability, and stable circular propagation. All these behaviors were observed in experiments.

Once the dye-doped liquid crystal is in the isotropic phase, the irradiation can be turned off, causing the nematic phase to invade the isotropic state. Surprisingly, the nematic to isotropic transition follows an entirely different course from the one described previously. The inverse process is characterized by the emergence of a foamlike texture of nematic bubbles as observed in Fig. 4(a). Subsequently, the bubbles start a coalescence process while shrinking, leading at the end to a labyrinthine texture that can remain for several minutes. Interestingly, Eq. (1) displays a similar dynamics when the parameter $C_{1}$ is abruptly decreased starting from a steady circular front [see Fig. 4(b)].

In brief, we have shown that light can induce finger instability in a liquid crystal photoisomerization experiment, leading to complex nematic-isotropic textures when the process is reversed. A simple phase-field model based on two reactiondiffusion equations for the order parameter and the cis-isomer of the azo dye reproduces all the observed behaviors. The (a)
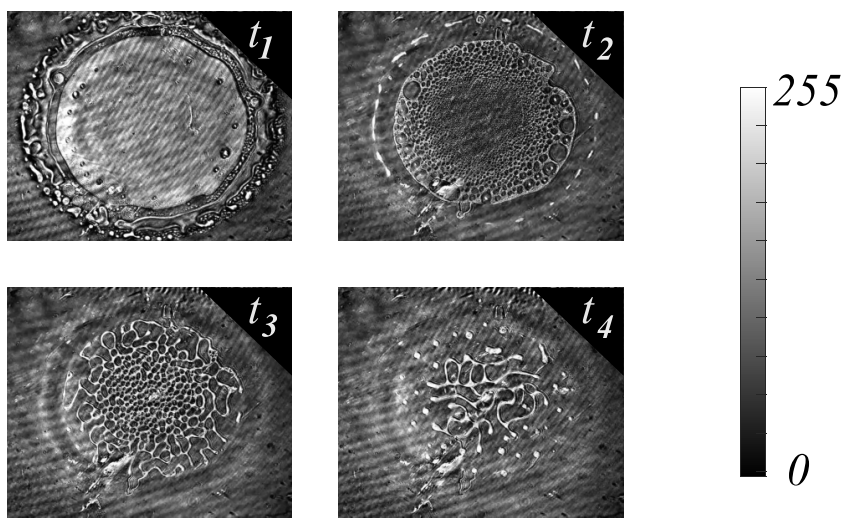

(b)
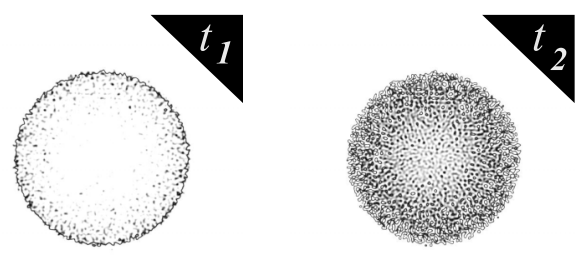

1
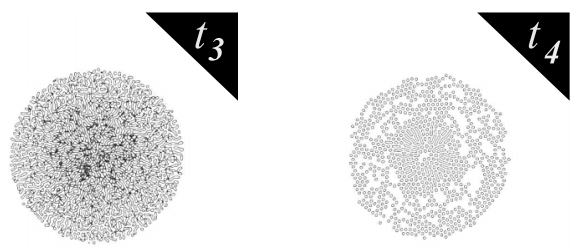

0

FIG. 4. Interface dynamics retraction for decreasing light intensity. (a) Temporal experimental snapshot sequence for the retraction of the isotropic phase when light intensity is decreased from $I_{\text {start }}=$ $0.7 \mathrm{~W}$ to $I_{\text {final }}=0.05 \mathrm{~W}$. The snapshot $t_{2}$ corresponds to a foamlike texture, while $t_{3}$ is a labyrinthine texture. (b) Temporal sequence of the evolution of $u$, considering Eq. (1) starting from an isotropic bubble, in which the $C_{1}$ parameter is abruptly decreased. The values of the parameters are $C_{0}=-1.0, C_{1}=0.3, \omega=115, D_{H}=-2.4$, $D_{I}=0.0$, and $\lambda=0.2$, and a two-dimensional grid with $600 \times 600$ points was chosen.

control over the fingers' average interdigital spacing allows for the possibility to light-steered fingering instabilities and for interface roughness control. Finally, we foresee these possibilities not only in the reversible photoisomerization process as shown in the experiments but also in permanent photoisomerizable polymerizations, where controlling the growth interface could lead to different textures of the final polymeric product.

The authors thank D. Escaff and M. Wilson for preliminary simulations. G.G.-C. acknowledges the financial support from ANID-PFCHA Doctorado Nacional Grant No. 2017 21171672. M.G.C. thanks ANID Millenium Science initiative program ICN17_012 and Fondecyt Project No. 1210353. 
[1] D. X. Viegas, Forest fire propagation, Philos. Trans. R. Soc. London, Ser. A 356, 2907 (1998).

[2] G. Sivashinsky, Diffusional-thermal theory of cellular flames, Combust. Sci. Technol. 15, 137 (1977).

[3] S. H. Tirmizi and W. N. Gill, Experimental investigation of the dynamics of spontaneous pattern formation during dendritic ice crystal growth, J. Cryst. Growth 96, 277 (1989).

[4] J. R. de Bruyn, Fingering Instability of Gravity Currents in Thin-Layer Electrochemical Deposition, Phys. Rev. Lett. 74, 4843 (1995).

[5] T. Niki, K. Muraoka, and K. Shimizu, Distribution of capillary nonperfusion in early-stage diabetic retinopathy, Ophthalmology 91, 1431 (1984).

[6] C. Giverso, M. Verani, and P. Ciarletta, Branching instability in expanding bacterial colonies, J. R. Soc., Interface 12, 20141290 (2015).

[7] G. M. Homsy, Viscous fingering in porous media, Annu. Rev. Fluid Mech. 19, 271 (1987).

[8] P. Saffman, Viscous fingering in Hele-Shaw cells, J. Fluid Mech. 173, 73 (1986).

[9] S. Hill, Channeling in packed columns, Chem. Eng. Sci. 1, 247 (1952).

[10] P. G. Saffman and G. I. Taylor, The penetration of a fluid into a porous medium or Hele-Shaw cell containing a more viscous liquid, Proc. R. Soc. London, Ser. A 245, 312 (1958).

[11] F. Box, O. Kodio, D. OKiely, V. Cantelli, A. Goriely, and D. Vella, Dynamic Buckling of an Elastic Ring in a Soap Film, Phys. Rev. Lett. 124, 198003 (2020).

[12] J. P. Sauppe, S. Palaniyappan, B. J. Tobias, J. L. Kline, K. A. Flippo, O. L. Landen, D. Shvarts, S. H. Batha, P. A. Bradley, E. N. Loomis, N. N. Vizirani, C. F. Kawaguchi, L. Kot, D. W. Schmidt, T. H. Day, A. B. Zylstra, and E. Malka, Demonstration of Scale-Invariant Rayleigh-Taylor Instability Growth in Laser-Driven Cylindrical Implosion Experiments, Phys. Rev. Lett. 124, 185003 (2020).

[13] T. Gao, M. Mirzadeh, P. Bai, K. M. Conforti, and M. Z. Bazant, Active control of viscous fingering using electric fields, Nat. Commun. 10, 4002 (2019).

[14] V. Odent, M. Clerc, C. Falcón, U. Bortolozzo, E. Louvergneaux, and S. Residori, Photo-isomerization fronts in dye-doped nematic liquid crystals, Opt. Lett. 39, 1861 (2014).

[15] V. Odent, E. Louvergneaux, M. G. Clerc, and I. Andrade-Silva, Optical wall dynamics induced by coexistence of monostable and bistable spatial regions, Phys. Rev. E 94, 052220 (2016).

[16] P.-G. De Gennes and J. Prost, The Physics of Liquid Crystals, International Series of Monographs on Physics, Vol. 83 (Oxford University Press, Oxford, 1993).

[17] I.-C. Khoo, Liquid Crystals, Wiley Series in Pure and Applied Optics, Vol. 64 (Wiley, Hoboken, NJ, 2007).

[18] S. Singh, Phase transitions in liquid crystals, Phys. Rep. 324, 107 (2000).

[19] I. Janossy, L. Csillag, and A. D. Lloyd, Temperature dependence of the optical Freedericksz transition in dyed nematic liquid crystals, Phys. Rev. A 44, 8410 (1991).

[20] I. Janossy and T. Kosa, Influence of anthraquinone dyes on optical reorientation of nematic liquid crystals, Opt. Lett. 17, 1183 (1992).
[21] T. Kosa, L. Sukhomlinova, L. Su, B. Taheri, T. J. White, and T. J. Bunning, Light-induced liquid crystallinity, Nature (London) 485, 347 (2012).

[22] M. Rumi, T. J. White, and T. J. Bunning, Phototropic guest-host liquid crystal systems: Environmental effects on naphthopyran kinetics, J. Phys. Chem. B 120, 12755 (2016).

[23] A. G. Chen and D. J. Brady, Real-time holography in azo-dyedoped liquid crystals, Opt. Lett. 17, 441 (1992).

[24] M. Chen, H. Dai, D. Wang, Y. Yang, D. Luo, X. Zhang, and C. Liu, Thermally and optically tunable lasing properties from dye-doped holographic polymer dispersed liquid crystal in capillaries, J. Appl. Phys. 123, 103105 (2018).

[25] V. Chigrinov, J. Sun, and X. Wang, Photoaligning and photopatterning: New LC technology, Crystals 10, 323 (2020).

[26] F. Derrien, J. Henninot, M. Warenghem, and G. Abbate, A thermal $(2 \mathrm{~d}+1)$ spatial optical soliton in a dye doped liquid crystal, J. Opt. A 2, 332 (2000).

[27] I. Andrade-Silva, U. Bortolozzo, C. Castillo-Pinto, M. Clerc, G. González-Cortés, S. Residori, and M. Wilson, Dissipative structures induced by photoisomerization in a dye-doped nematic liquid crystal layer, Philos. Trans. R. Soc. A 376, 20170382 (2018).

[28] I. Andrade-Silva, U. Bortolozzo, M. G. Clerc, G. GonzálezCortés, S. Residori, and M. Wilson, Spontaneous light-induced Turing patterns in a dye-doped twisted nematic layer, Sci. Rep. 8, 12867 (2018).

[29] K. Katayama, D. Kato, K.-i. Nagasaka, M. Miyagawa, W. Y. Sohn, and K.-W. Lee, Origin of optical nonlinearity of photoresponsive liquid crystals revealed by transient grating imaging, Sci. Rep. 9, 5754 (2019).

[30] C. Manzo, D. Paparo, L. Marrucci, and I. Janossy, Lightinduced rotation of dye-doped liquid crystal droplets, Phys. Rev. E 73, 051707 (2006).

[31] E. Brasselet and S. Juodkazis, Optical angular manipulation of liquid crystal droplets in laser tweezers, J. Nonlinear Opt. Phys. Mater. 18, 167 (2009).

[32] S. Bono, S. Sato, and Y. Tabe, Unidirectional rotation of cholesteric droplets driven by UV-light irradiation, Soft Matter 13, 6569 (2017).

[33] Y. Sakai, W. Y. Sohn, and K. Katayama, Optical motion control of liquid crystalline droplets by host-guest molecular interaction, Soft Matter 15, 7159 (2019).

[34] Y. Sakai, W. Y. Sohn, and K. Katayama, Photo-controllable rotational motion of cholesteric liquid crystalline droplets in a dispersion system, RSC Adv. 10, 21191 (2020).

[35] D.-K. Yang and S.-T. Wu, Fundamentals of Liquid Crystal Devices (Wiley, Hoboken, NJ, 2014).

[36] G. Zimmerman, L.-Y. Chow, and U.-J. Paik, The photochemical isomerization of azobenzene, J. Am. Chem. Soc. 80, 3528 (1958).

[37] I. Janossy, Optical reorientation in dye-doped liquid crystals, J. Nonlinear Opt. Phys. Mater. 8, 361 (1999).

[38] I. Khoo, P. Chen, M. Shih, A. Shishido, S. Slussarenko, and M. Wood, Supra optical nonlinearities (SON) of methyl red-and azobenzene liquid crystal-doped nematic liquid crystals, Mol. Cryst. Liq. Cryst. Sci. Technol., Sect. A 358, 1 (2001). 
[39] D. Statman and I. Janossy, Study of photoisomerization of azo dyes in liquid crystals, J. Chem. Phys. 118, 3222 (2003).

[40] P. Oswald, J. Bechhoefer, and A. Libchaber, Instabilities of a Moving Nematic-Isotropic Interface, Phys. Rev. Lett. 58, 2318 (1987).
[41] P. Oswald, J. Bechhoefer, and F. Melo, Pattern formation during the growth of liquid crystal phases, MRS Bull. 16, 38 (1991).

[42] J. McLean and P. Saffman, The effect of surface tension on the shape of fingers in a Hele Shaw cell, J. Fluid Mech. 102, 455 (1981). 